\title{
Anorexia nervosa in pregnancy: a case report and review of the literature
}

\author{
K Dinas, A Daniilidis MD, K Sikou SHO, T Tantanasis, S Kasmas SHO and J Tzafettas
}

Department of Obstetrics-Gynaecology, Hippokratio University Hospital, Thessaloniki, Greece

\begin{abstract}
Summary: Anorexia nervosa is a complex illness rarely encountered in pregnant women. It is a disorder characterized by markedly decreased food intake accompanied by a distorted body image, resulting in an inability to maintain the body weight within $85 \%$ of ideal body weight. We describe a case of a pregnant woman diagnosed with anorexia nervosa at 28 weeks of gestation. Her body mass index was $17 \mathrm{~kg} / \mathrm{m}^{2}$. A live male infant weighing 2,08 kg was delivered prematurely via vaginal delivery at 35 weeks of gestation. Pregnant women with anorexia nervosa may have a higher risk of hypertension, miscarriage, difficult labour, premature delivery and intrauterine growth restriction. Management of pregnancy complicated with anorexia nervosa requires involvement of a multidisciplinary team and hospitalization in severe cases.
\end{abstract}

Keywords: anorexia nervosa and pregnancy, IUGR, premature delivery

\section{INTRODUCTION}

Anorexia nervosa is a complex illness with characteristic psychological and physical abnormalities. Although gynaecologists are often the first physicians consulted because of menstrual disorders, the syndrome is rarely encountered in pregnant women. We describe a case of a pregnant woman diagnosed with anorexia nervosa who was hospitalized in our clinic and delivered prematurely via vaginal delivery at 35 weeks gestation. At delivery her live male infant was small for dates, weighing 2,08 kg, had Apgar scores $7 / 1$ and 9/5 and an uneventful postpartum course. The mother's anorexia remained unchanged postpartum.

\section{CASE}

Mrs MK was a 23-year-old primigravida who presented to the obstetric emergency unit with a history of contractions. She was 28 weeks pregnant as determined by her last menstrual period and transvaginal ultrasound at nine weeks. She was a poor attendant at the outpatient antenatal clinic. On assessment, she was found to be in premature labour with contractions and cervical dilation of $3 \mathrm{~cm}$. She was feeling unwell, weak, unable to walk without assistance and seemed obviously underweight. Cardiotocographic (CTG) assessment was satisfactory and she was administered tocolytics according to our protocol for preterm labour (indomethacin per rectum + ritodrine intravenously and betamethazone) and she was transferred to our high-risk pregnancy unit. Contractions stopped after two hours. She underwent extensive medical evaluation which showed microcytic hypochromic anaemia and a body

Correspondence to: A Daniilidis, 9 Smirnis, 56224 Evosmos, Thessaloniki, Greece

Email: ange1972@otenet.gr weight of $40 \mathrm{~kg}$. She was $155 \mathrm{~cm}$ tall and her body mass index (BMI) was $17 \mathrm{~kg} / \mathrm{m}^{2}$. Psychiatric evaluation concluded that she had both anorexia nervosa and depression, thought to be related to a stressful family environment. Her husband was unemployed, and he admitted to being dissatisfied with the marriage and confessed to having an affair a year earlier with a mutually close female friend.

During her hospitalization, she was managed by a team which included dieticians and physicians. She received intravenous fluids, oral iron supplementations and multivitamins. After considerable effort, she was provided with approximately 1000-1200 calories of daily intake. The fetal wellbeing was assessed each week by growth, Doppler studies and with CTG. The fetus was symmetrically small for dates without any other worrisome signs. The decision was made to continue with the pregnancy under intense surveillance for as long as possible. After almost seven weeks of hospitalization, she reached 35 weeks of gestation and delivered a live male infant weighing 2,08 kg and with Apgar scores of $7 / 1$ and $5 / 9$, normally after spontaneous onset of labour. The infant was transferred initially to the neonatal intensive care unit for observation but was discharged within 24 hours. The mother's weight was $39 \mathrm{~kg}$ immediately after delivery. There were no postpartum complications, but her anorexia nervosa persisted without any improvement despite the efforts of our multidisciplinary team.

\section{COMMENT}

In a normal pregnancy the average weight gain from before conception to shortly before delivery is about $12.5 \mathrm{~kg}$. ${ }^{1}$ In western countries, the recommended daily amount of energy during pregnancy is $2400 \mathrm{kcal}$ - about $250-300 \mathrm{kcal}$ more than the nonpregnant requirement. ${ }^{1}$ Anorexia nervosa is a disorder characterized by markedly decreased food intake, a phobia of gaining 
weight and distorted body image, resulting in an incapacity to maintain the body weight within $85 \%$ of ideal body weight. ${ }^{2}$ It is now recognized that there is a spectrum of psychosomatic dysfunction from anorexia nervosa to bulimia, often associated with menstrual disturbances.

The prevalence of anorexia nervosa in young women is about $0.5 \%$, but the prevalence of eating disorders in women in general is not known. ${ }^{3}$ The course may be episodic or chronic with full recovery in $40 \%$ and another $30 \%$ with significant improvement. In about $20 \%$ of cases women remain ill and there is a $5 \%$ mortality rate. ${ }^{4,5}$ Associated physiological signs which increase maternal morbidity and mortality may include hypothermia, bradycardia, hypotension, oedema and other metabolic changes. There is a higher risk of hypertension, miscarriage and difficult labour. ${ }^{6}$ Perinatal morbidity and mortality is also reported to be higher in anorexic women owing to an increased rate of premature delivery and intrauterine growth restriction (IUGR). Underweight status before pregnancy and inadequate weight gain during the second half of pregnancy independently increases the risk of preterm labour and IUGR. ${ }^{7}$ A critical issue is the relationship between the diet of the mother and the wellbeing of the fetus. Studies of caloric restriction during pregnancy have indicated a lesser maternal weight gain at the expense of lighter birth weights. ${ }^{8,9}$ Women with anorexia nervosa are 2.3 times more likely to deliver infants of low birth weight and 1.5 times more likely to have a fetal death. ${ }^{9,10}$ In fact, there is a statistically linear relationship between prepregnancy body mass and birth weight, as well as between prenatal weight gain and birth weight. ${ }^{11}$ Also, weight gain during pregnancy from the first trimester in underweight women may advance and promote a rise in maternal weight of more than $6 \mathrm{~kg}$ by the 28th week of pregnancy with a high probability that the rest of the pregnancy will progress normally. ${ }^{12}$ A recent study included 302 women hospitalized with eating disorders before pregnancy and tried to associate the prepregnancy status and the birth weight of infants as well as prematurity. The result was that low birth weight and prematurity may in fact be due to low maternal weight at conception, not to anorexia nervosa per se. ${ }^{13}$ Interestingly, another study reported that women with previous anorexia nervosa who were normal weight at the time of conception did not have infants that were low birth weight or premature. ${ }^{14}$ In addition there is evidence to suggest that a pregnancy complicated by a very low birth weight baby may be associated with an increased risk of cardiovascular disease, obstructive lung disease and other problems in adult life. ${ }^{15}$ Those women known to have anorexia nervosa may deteriorate as pregnancy advances and after pregnancy rapid deterioration usually follows. ${ }^{16,17}$ The seriousness of the problem is worsened if presentation is preconception or in the first trimester of pregnancy. It is suggested that in order to prevent preterm labour and IUGR, a combined effort is required as early as possible prior to conception. ${ }^{16,17}$ Underweight women who undergo ovulation induction usually do not reach the adequate weight gain despite counselling. It is currently recommended that ovulation induction be delayed and reliable contraception offered to these women until their weight is at least above $45 \mathrm{~kg}{ }^{18}$ Since the majority of cell division occurs during the first trimester, it is essential that the nutritional status is optimized before conception. ${ }^{18}$

Management of pregnancy complicated with anorexia nervosa requires involvement of a multidisciplinary team of obstetricians, medicine specialists, nutritionists, psychiatrists, neonatologists and hospitalization when indicated in severe cases. Expert consultation and careful monitoring of maternal weight gain and fetal surveillance is essential in order to achieve the best outcome for the pregnancy.

\section{REFERENCES}

1 Truswell S. ABC of Nutrition. Nutrition for pregnancy. Br Med J 1985;291:263-6

2 Walsh JM, Wheat ME, Freund K. Detection, evaluation, and treatment of eating disorders: the role of the primary care physician. J Gen Intern Med 2000;15:577

3 Haessler A, Rosenthal M. Psychologic aspects of gynecology. Anorexia nervosa. Curr Obstet Gynecol Diagn Treat 2003;59:1068

4 Lingam R, McCluskey S. Eating disorders associated with hyperemesis gravidarum. J Psychosom Res 1996;40:231-4

5 Micali N, Treasure J, Simonoff E. Eating disorders symptoms in pregnancy: a longitudinal study of women with recent and past eating disorders and obesity. J Psychosom Res 2007;63:297-303

6 Kumar R, O'Dowd L. Psychiatric problems in pregnancy and the puerperium. Eating disorders. Turnbull's Obstet 2002;19:302

7 Siega-Riz AM, Adair LS, Hobel CJ. Maternal underweight status and inadequate rate of weight gain during the third trimester of pregnancy increases the risk of preterm delivery. J Nutr 1996;126:146-153

8 Koubaa S, Hallstrom T, Lindholm C, Hirschberg AL. Pregnancy and neonata outcomes in women with eating disorders. Obstet Gynecol 2005;105:255-60

9 Campell DM, MacGillivray I. The effect of a low calorie diet or a thiazide diuretic on the incidence of preeclampsia and on birthweight. $\mathrm{Br}$ J Obstet Gynecol 1975;82:572

10 Rush D. Effects of changes in protein and calorie intake during pregnancy on the growth of the human fetus. In: Calmers I, Enkin M, Keirse MJNC, eds. Effective Care in Pregnancy and Childbirth. Oxford: Oxford University Press, 1989:255

11 Johnson AA, Knight EM, Edwards CH, et al. Dietary intakes, anthropometric measurements and pregnancy outcomes. J Nutr 1994;124:936

12 Myers SA, Ferguson R. A population study of the relationship between fetal death and altered fetal growth. Obstet Gynecol 1989;74:325

13 Sollid CP, Wisborg K, Hjort J, Secher NJ. Eating disorder that was diagnosed before pregnancy and pregnancy outcome. Am J Obstet Gynecol 2004;190:206-10

14 Ekeus C, Olausson PO, Hjern A. Psychiatric morbidity is related to parental age: a national cohort study. Psychol Med 2006;36:269-76

15 Baker DJP. The fetal and infant origins of adult disease. Br Med J 1990;301:111

16 Woodside DB, Shekter-Wolfson LF. Parenting by parents with anorexia nervosa and bulimia nervosa. Int J Eating Dis 1990;9:303

17 Speroff L, Glass R, Kase N. Amenorrhea. Eating disorders and pregnancy. Clin Gynecol Endocrinol Infertil 1999;11:469-72

18 Balen A, Jacobs H. Anovulatory infertility and ovulation induction. Weight-related amenorrhea. Infertil Pract 2003;6:138-42 